\title{
GYG1 causing progressive limb girdle myopathy with onset during teenage years (Polyglucosan Body Myopathy 2)
}

Mahalekshmi Desikan ${ }^{1 *}$, Renata Siciliani Scalco ${ }^{1,2 *}$, Andreea Manole ${ }^{1}$, Alice R. Gardiner ${ }^{1}$, Anthony H. Schapira ${ }^{1}$, Robin H Lachmann ${ }^{3}$, Henry Houlden ${ }^{1}$, Janice L. Holton ${ }^{1}$, Rahul Phadke $^{1}$, Ros Quinlivan ${ }^{1}$

* Both authors contributed equally to this publication

1- MRC Centre for Neuromuscular Disease and Division of Neuropathology, UCL Institute of Neurology, National Hospital for Neurology and Neurosurgery, London, $U K$

2- Capes Foundation, Ministry of Education, Brazil

3- Charles Dent Metabolic Unit, National Hospital for Neurology and Neurosurgery, Queen Square, London, United Kingdom

\section{Authors Contribution:}

MD, literature review, interpretation of data, manuscript draft

RSS, study concept and design, acquisition of data, analysis and interpretation of data, manuscript draft

AGG, study concept and design, laboratory work, analysis and interpretation of data

AM, manuscript draft

AS and RHL acquisition of data, critical revision of manuscript for intellectual content

JLH and RP, histopathological analysis, analysis and interpretation of data, critical revision of manuscript for intellectual content

$\mathrm{RP}$, figure production

RQ, study concept and design, acquisition of data, analysis and interpretation of data, study supervision, critical revision of manuscript for intellectual content

\section{Corresponding Author:}

Dr Renata S. Scalco

8-11 Queen Square, WC1N 3BG, London UK

r.scalco@ucl.ac.uk 
Abstract: An 84-year-old lady with slowly progressive limb and axial muscle weakness with onset in her teens was referred for genetic investigations. Targeted next generation sequencing (NGS) revealed a homozygous mutation GYG1 in exon5:c.487delG:p.D163fs, confirming the diagnosis of Polyglucosan Body Myopathy 2 (PGBM2). Retrospective review of muscle pathology revealed a florid vacuolar myopathy with histochemical and ultrastructural features consistent with a polyglucosan storage myopathy. No cardiac symptoms were reported. Our case is consistent with the core phenotype of GYG1-related PGBM2 apart from an early onset of weakness without cardiac symptoms. The presence of $\alpha$ amylase resistant PAS-positive material in skeletal muscle biopsy of patients with slowly progressive limb girdle muscle weakness should prompt the search for GYG1 mutations. This case highlights the combined role of muscle pathology and NGS in the molecular resolution of undiagnosed patients with undiagnosed neuromuscular conditions.

\title{
Key Words:
}

Glycogen Storage Disease, polyglucosan body myopathy, progressive muscle weakness, limb girdle muscle weakness

\begin{abstract}
Abbreviations: CK: Creatinine Kinase; ECG: Electrocardiogram; FFPE: Formalin-fixed and paraffin-embedded; GSDs: Glycogen Storage Disorders; GSDXV: Glycogen Storage Disease type XV; PGBM2: Polyglucosan Body Myopathy 2; NGS: Next Generation Sequencing; Limb Girdle Muscular Dystrophy (LGMD)
\end{abstract}

\section{Introduction:}

Glycogen is a highly branched polymer of glucose residues, linked by $\alpha-(1,4)-$ and $\alpha$ $(1,6)$-glycosidic bonds and its synthesis involves a series of co-ordinated reactions catalysed 
by various enzymes. Pathogenic mutations in genes that encode enzymes involved in glycogen synthesis and degradation cause abnormal glycogen accumulation within a variety of cells to include skeletal and cardiac muscle, liver, and others (Glycogen Storage Disorders - GSDs). Related symptoms may include muscle weakness, hepatic dysfunction and cardiomyopathy.

The GYG1 gene encodes the skeletal muscle isoform of glycogenin (glycogenin-1), a protein which plays an important role in the biosynthesis of glycogen. Glycogenin-1 acts as a glycosyl transferase to catalyse synthesis of short, 7-13 residue glucose polymers from UDPglucose by a process of auto-glycosylation of a tyrosine residue. This is followed by elongation and branching of the glucose primer chains by glycogen synthase and branching enzymes, encoded by GYS1 gene in muscle and GBE1 genes respectively, to produce glycogen. Mutations in GYG1 have been shown to result in reduced, absent or functionally abnormal glycogenin-1, leading to defective priming of glycogen synthesis, glycogen depletion in skeletal muscle and abnormal glycogen accumulation in cardiac muscle (Glycogen Storage Disease type XV - GSDXV).(1,2)

Polyglucosan bodies consist of aggregates of abnormal amylopectin like polysaccharides with less branching than normal glycogen and variable degree of resistance to $\alpha$-amylase digestion. Seen as fibrillar structures on electron microscopy, their accumulation in muscle has been described in disorders of glycogen metabolism (GSDIV, VII and XV), AMP- activated protein kinase deficiency, $R B C K 1$-related disease and Lafora body disease.(2) Polyglucosan body accumulation with associated slowly progressive proximal myopathy has been recently reported with a recurring intronic GYGl homozygous mutation $(\mathrm{c}, 143+3 \mathrm{G}>\mathrm{C}) .(3,4)$ Homozygous or compound heterozygous mutations in $G Y G 1$ may cause Polyglucosan Body Myopathy 2 (PGBM2) (OMIM \#616199). The clinicopathological spectrum of $G Y G 1$-disease is expanding, to include distal myopathy. We report a 
patient with progressive muscle weakness and a late diagnosis of $G Y G 1$-associated PGBM2 established by Next Generation Sequencing (NGS).

\section{Case Report}

An 84-year-old lady with a history of chronic and slowly progressive muscle weakness with onset in her teens, affecting axial, proximal and distal limb muscles in an asymmetrical distribution, was referred for diagnostic investigations. She had a normal childhood and was good at sport. She used to do ballet and tap dance until the age of 15 years when she began to notice that she could not "hurry" anymore as her agility was reduced. She reported proximal muscle weakness from 15 years of age, initially more evident in her lower limbs. Symptoms gradually progressed and by 40 years of age she had difficulty climbing stairs and in her 50s she had to move to a ground floor flat. She was unable to mobilise without a rollator at the age of 70 years, which she used for the last 15 years. The last two years were particularly more difficult as weakness limited her daily activities including showering and cooking. She gradually noticed difficulties opening cans and bottles due to distal hand weakness which developed over many years, but she could not recall the exact age of onset. In the 1950s, when she was in her 20s she was given a clinical diagnosis of limb girdle muscular dystrophy.. Throughout her life, she had no symptoms of myoglobinuria, cardiac, respiratory or gastrointestinal involvement. Her family history was unremarkable for neuromuscular diseases and she had no offspring.

A quadriceps muscle biopsy was performed 30 years ago, when she was 58 years old to exclude polymyositis because she developed transient myalgia and tenderness around the shoulder girdle muscles. According to the clinical synopsis in the biopsy report: CK was 122 
IU/L. The biopsy showed a vacuolar myopathy with accumulation of PAS-positive material leading to a diagnosis of an uncertain form of GSD.

The patient requested a referral to our tertiary centre in her 80 s for a comprehensive review and further investigation, given the rapid developments in the field of neuromuscular disease since the 1990s. On physical examination at the age of 84 , there was evidence of global muscle atrophy, moderate bilateral scapular winging and asymmetrical weakness in the axial and limb muscles (Table 1). Cranial musculature was spared. There were no cardiac symptoms or abnormal clinical signs on examination.

\begin{tabular}{|l|c|c|}
\hline & Left & Right \\
\hline $\begin{array}{l}\text { Neck muscles } \\
\text { (Flexion, Extension, Rotation) }\end{array}$ & 4 & 4 \\
\hline Trapezius & 4 & 4 \\
\hline Deltoid & 2 & 2 \\
\hline Biceps & 3 & 2 \\
\hline Triceps & 4 & 3 \\
\hline Pronation / Supination & $3 / 3$ & $4 / 4$ \\
\hline Flexion / Extension at Wrist & $3 / 2$ & $4 / 3$ \\
\hline Finger Flexion / Extension & $4 / 3$ & $5 / 3$ \\
\hline Abduction of the Fingers & 3 & 3 \\
\hline Hip Flexion / Extension & $3 / 4$ & $3 / 4$ \\
\hline Leg Flexion / Extension & $3 / 3$ & $3 / 4$ \\
\hline Dorsiflexion of the Feet & 3 & 3 \\
\hline Plantar Flexion & 4 & 4 \\
\hline
\end{tabular}

Table 1: MRC Score. Physical exam performed at the age of 84 .

Serum creatine kinase (CK) was essentially normal (CK: 147 IU/L, normal range 26140). Random blood glucose and serum lactate were normal. Erythrocyte glycogen content was reported to be low at $4.9 \mu \mathrm{g} / \mathrm{g}$ of haemoglobin (10-120) on an initial sample, but was low normal on subsequent testing $(10 \mu \mathrm{g} / \mathrm{g})$. Enzyme analysis (blood sample) for acid alpha glucosidase, phosphorylase $\mathrm{b}$ kinase and beta galactosidase was normal. Neurophysiology 
studies showed myopathic features. ECG was within normal limits. The patient declined to have a cardiac echo.

Genetic investigation by NGS Illumina "TruSight One" enrichment panel designed to screen for 60 relevant genes associated with rhabdomyolysis (mostly reviewed on (5)) was performed as it covered all GSD related genes. The rhabdomyolysis panel confirmed GYG1 homozygous mutation in exon 5 (c.487delG:p.D163fs). The change was an aspartic acid to a threonine at amino acid position 163 and a shift in the reading frame, resulting in a premature stop codon at position 167 (D163Tfs*5). This mutation was present in the control population but only in the heterozygous state (57 out of 120,474) and not as a homozygote and, predominantly in North European individuals $(52 / 66,388)$. This concurs with the lack of family history and the presence of this change in a non-consanguineous pedigree. The frequency of heterozygotes suggests that LGMD-like phenotype due to this mutation may not be that rare in the European population.

Following the genetic diagnosis, we tried to retrieve the historical biopsy performed many decades ago in a different hospital in order to review the muscle pathology. Unfortunately, the original stained slides could not be traced. A single formalin-fixed and paraffin-embedded (FFPE) block was identified and retrieved for work-up (Figure 1). Sections were cut and stained from the archival paraffin block. Staining for PAS and PAS with diastase digestion was performed as per previously described protocols.(6) Immunostaining was performed using antibodies against fast myosin (Leica Microsystems (Novocastra), WB-MHCf), slow myosin (Leica Microsystems (Novocastra), WB-MHCs), ubiquitin (Santa-Cruz, SC-8017) and p62 (Abcam ab56416).

The biopsy showed marked myopathic changes including patchy, asymmetric fatty infiltration within fascicles and increased fibre size variation. There was overall slow fibre 
predominance with preferential fast fibre atrophy. Over $80 \%$ of fibres appeared vacuolated, containing polymorphic subsarcolemmal and internal basophilic inclusions that were intensely PAS-positive. The inclusions ranged from discrete large 'lakes' to myriad smaller deposits to fine 'dust-like' material. Fibres cut longitudinally revealed skip lesions. Virtually all of this material was resistant to $\alpha$-amylase digestion in keeping with polyglucosan accumulation, and many such fibres appeared depleted of glycogen. The storage material was also immunoreactive for desmin and ubiquitin. CD68 labeling was very sparse and unrelated to the vacuolar storage. A region of interest marked on the Haematoxylin and Eosin stained section was mapped to and cut out from the paraffin block, and re-processed for electron microscopy. Although preservation was sub-optimal, presence of fibrillar polyglucosan storage material was confirmed.

\section{Discussion}

To date, only a few patients with GYG1 mutations and a neuromuscular phenotype are reported. In 2010, Moslemi et al (7) first described a young male with muscle weakness, cardiomyopathy and cardiac arrhythmia caused by bi-allelic mutations in GYG1. Deltoid muscle biopsy of this patient showed glycogen depletion in skeletal muscle, but accumulation of PAS-positive material in cardiac myocytes. The exact nature of the PAS-positive material in the reported patient could not be established. Subsequently, Malfatti et al (4) presented 7 patients with a slowly progressive skeletal myopathy of juvenile or adult onset with polyglucosan bodies on muscle biopsy, but no cardiac involvement. DNA analysis revealed homozygous or compound heterozygous mutations in GYG1 gene and the authors suggested that the varying phenotypes are probably related to the type of $G Y G 1$ pathogenic variant. In our case, the only material available for pathological reassessment was a FFPE block. In 
contrast to the pathology of cases described by Malfatti et al, the PAS+ inclusions in our patient's biopsy were strongly $\alpha$-amylase resistant, and there was variable glycogen depletion in fibres containing polyglucosan. The true extent of glycogen depletion in our case is difficult to ascertain due to the nature of the biopsy material being formalin fixed and paraffin processed, and at least part of the glycogen depletion may relate to the processing effect. As there was no record of previous electron microscopic examination, we re-processed a region of interest from the paraffin embedded tissue for electron microscopy, and successfully demonstrated fibrillar storage material in keeping with polyglucosan, albeit of an inferior quality to freshly processed tissue for electron microscopy. Our case demonstrates the utility of this technique for ultrastructural assessment of structural abnormalities or storage material from cases where no other tissue except FFPE may be available. The slow fibre predominance and fast fibre atrophy may suggest a metabolic adaptation to the defective glycogen metabolism.

Muscle MRI in individual cases has shown preferential involvement of deltoids and the gluteal muscles and could be used as a non-invasive tool to characterise the pattern of muscle involvement and monitor progression. $(8,9)$ Akman et al. reported five adult patients with polyglucosan myopathy from four unrelated Sardinian families, all with the same autosomal recessive GYG1 mutation. Taking into consideration all published case reports, the most common mutation described to date is a single nucleotide substitution at donor splice site in intron 2 c. $143+3 \mathrm{G}>$ C. $(2,4,7,10)$ Majority of PGBM2 patients described to date developed muscle weakness in adulthood, as opposed to our patient who presented symptoms since her teens.(11) Even though late onset myopathy with progressive symmetrical limb girdle weakness is the most common muscle phenotype; early onset, facial muscle weakness, asymmetry of limb muscle weakness and distal limb weakness (as described in our case), myalgia, exercise intolerance and fluctuation in symptoms have all been reported. $(3,4,8,9)$ 
A purely cardiac phenotype in 3 patients has been published by Oldfors et al in 2017 (12), with the genetic defect being c.304G > C, p.Asp102His mutation in exon 3 of the GYG1 gene. The authors think that cardiomyopathy and cardiac failure relate to cellular overload of polyglucosan material, leading to cell death and secondary fibrosis.

The pathogenesis of polyglucosan body accumulation in GYG1 myopathy is not fully understood, but is thought to be related to ubiquitin-proteasomal and autophagic degradation pathways.(10) Many of the polyglucosan inclusions in the reported case labeled with ubiquitin, suggesting protein accumulation and activation and/or overloading of the ubiquitinproteosomal pathway.

Our case describes a patient with progressive muscle weakness who was originally diagnosed with LGMD and subsequently polymyositis based on her initial clinical presentation. Later on, following muscle biopsy, her diagnosis was modified to 'an uncertain form of GSD'. The genetic diagnosis of GYG1-related PGBM2 was established by targeted NGS, almost 70 years after disease presentation, highlighting the importance of NGS as a diagnostic tool when assessing undiagnosed patients presenting with muscle weakness. Our case extends the clinical phenotype of this disease to presentation in teens with slow progression over many years. A comprehensive muscle biopsy review was undertaken from a block of paraffin embedded tissue including D-PAS and p62 staining following the genetic diagnosis. The findings were consistent with a polyglucosan storage myopathy, reaffirming the genetic diagnosis. The original tissue diagnosis of an 'uncertain form of GSD' may reflect the fact that PAS staining with diastase digestion was not performed, and there was no ultrastructural assessment of the substantial storage material evident on light microscopy. Our case highlights the need for routine inclusion for diastase-PAS in the histochemical panel for diagnostic muscle biopsies. In the context of NGS-based approach becoming increasingly established for resolving molecularly unconfirmed cases, our case represents a good example 
of 'reverse pathology' where any type of archival material when available should be reviewed to ascertain compatibility with the genetic diagnosis, and/or extension of known pathological phenotypes associated with a particular known disease causing gene. Unfortunately we were unable to perform a detailed cardiac investigation with echocardiography or cardiac MRI on our patient as she declined the investigations due to travelling difficulties as a consequence of her muscle impairment. In the absence of cardiac symptoms, normal cardiovascular system on bedside examination, normal ECG and longevity we presume that she had a pure skeletal muscle phenotype, with no significant cardiac involvement. Further studies should be performed to understand the use of erythrocyte glycogen content as a potential screening test in this group of patients.

\section{Acknowledgements}

This research was funded by NHS England, and supported in part be by the National Institute for Health Research University College London Hospitals Biomedical Research Centre.

Patient provided signed consent form for case report publication.

\section{REFERENCES}

1. Roach PJ, Depaoli-Roach AA, Hurley TD, Tagliabracci VS. Glycogen and its metabolism: some new developments and old themes. Biochemical J 2012;441(3):763-87.

2. Hedberg-Oldfors C, Oldfors A. Polyglucosan storage myopathies. Mol Aspects Med 2015;46:85-100.

3. Akman HO, Aykit Y, Amuk OC, Malfatti E, Romero NB, Maioli MA, et al. Late-onset polyglucosan body myopathy in five patients with a homozygous mutation in GYG1. Neuromuscul Disord 2016;26(1):16-20.

4. Malfatti E, Nilsson J, Hedberg-Oldfors C, Hernandez-Lain A, Michel F, Dominguez-Gonzalez C, et al. A new muscle glycogen storage disease associated with glycogenin-1 deficiency. Ann Neurol 2014;76(6):891-8.

5. Scalco RS, Gardiner AR, Pitceathly RD, Zanoteli E, Becker J, Holton JL, et al. Rhabdomyolysis: a genetic perspective. Orphanet J Rare Dis 2015;10:51.

6. Bernfeld P. Enzymes of starch degradation and synthesis. Adv Enzymol Relat Subj Biochem 1951;12:379-428. 
7. Moslemi AR, Lindberg C, Nilsson J, Tajsharghi H, Andersson B, Oldfors A. Glycogenin-1 deficiency and inactivated priming of glycogen synthesis. N Eng J Med 2010;362(13):1203-10.

8. Colombo I, Pagliarani S, Testolin S, Cinnante CM, Fagiolari G, Ciscato P, et al. Longitudinal follow-up and muscle MRI pattern of two siblings with polyglucosan body myopathy due to glycogenin-1 mutation. J Neurol Neurosurg Psychiatry 2016;87(7):797-800.

9. Luo S, Zhu W, Yue D, Lin J, Wang Y, Zhu Z, et al. Muscle pathology and whole-body MRI in a polyglucosan myopathy associated with a novel glycogenin-1 mutation. Neuromuscul Disord 2015;25(10):780-5.

10. Fanin M, Torella A, Savarese M, Nigro V, Angelini C. GYG1 gene mutations in a family with polyglucosan body myopathy. Neurol Genet 2015;1(3):e21.

11. Hedberg-Oldfors C, Mensch A, Visuttijai K, Stoltenburg G, Stoevesandt D, Kraya T, et al. Polyglucosan myopathy and functional characterization of a novel GYG1 mutation. Acta Neurol Scand 2017.

12. Hedberg-Oldfors $C$, Glamuzina E, Ruygrok $P$, Anderson $L$, Elliott $P$, Watkinson $O$, et al. Cardiomyopathy as presenting sign of glycogenin-1 deficiency-report of three cases and review of the literature. J Inherit Metab Dis 2017;40(1):139-49.

Figure 1. Quadriceps muscle biopsy performed at 58 years of age; only paraffin-embedded tissue was available for re-analysis. There is marked myopathic variation in fibre size (A, haematoxylin \& eosin). Several fibres appear vacuolated, containing subsarcolemmal and internal basophilic inclusions that are intensely PAS-positive (B) and a majority are strongly $\alpha$-amylase resistant consistent with polyglucosan storage (C). Many fibres containing polyglucosan material show partial glycogen depletion. Ultrastructural examination of the reprocessed paraffin-embedded material confirms electron-dense fibrillary polyglucosan storage (D, higher magnification inset). Scale bar in A-C $=100$ microns. 

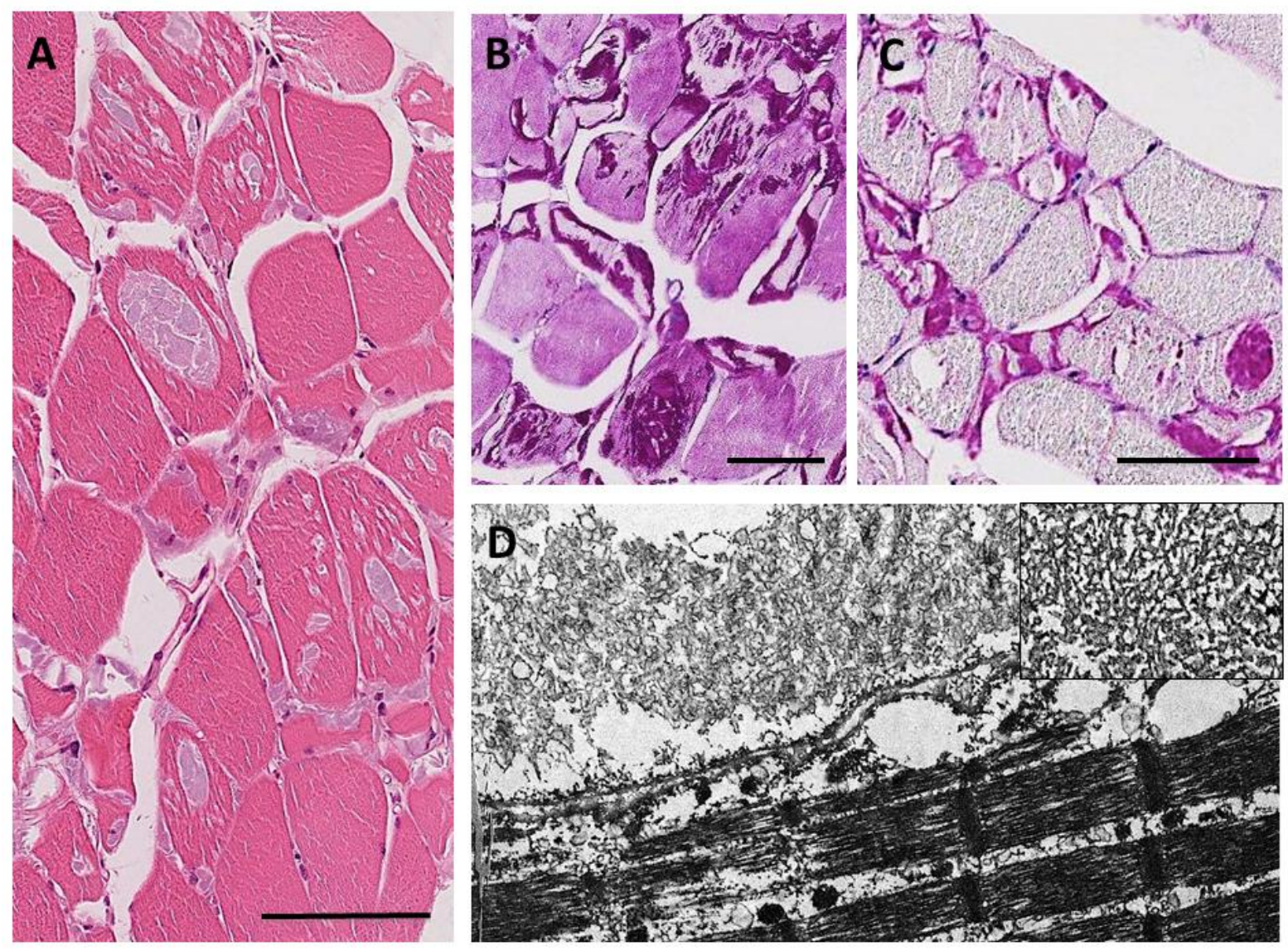